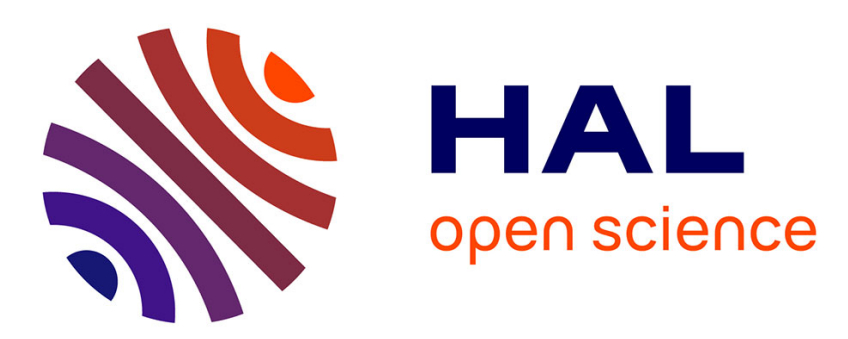

\title{
A new device for combined Coulomb excitation and isomeric conversion electron spectroscopy with fast fragmentation beams
}

E. Clément, A. Görgen, W. Korten, A. Bürger, A. Chatillon, Y. Le Coz, Ch. Theisen, M. Zielinska, Bertram Blank, P.J. Davies, et al.

\section{To cite this version:}

E. Clément, A. Görgen, W. Korten, A. Bürger, A. Chatillon, et al.. A new device for combined Coulomb excitation and isomeric conversion electron spectroscopy with fast fragmentation beams. Nuclear Instruments and Methods in Physics Research Section A: Accelerators, Spectrometers, Detectors and Associated Equipment, 2008, 587, pp.292-299. 10.1016/j.nima.2008.01.059 . in2p3-00189007

\section{HAL Id: in2p3-00189007 https://hal.in2p3.fr/in2p3-00189007}

Submitted on 19 Nov 2007

HAL is a multi-disciplinary open access archive for the deposit and dissemination of scientific research documents, whether they are published or not. The documents may come from teaching and research institutions in France or abroad, or from public or private research centers.
L'archive ouverte pluridisciplinaire HAL, est destinée au dépôt et à la diffusion de documents scientifiques de niveau recherche, publiés ou non, émanant des établissements d'enseignement et de recherche français ou étrangers, des laboratoires publics ou privés. 


\title{
A New Device for Combined Coulomb Excitation and Isomeric Conversion Electron Spectroscopy with Fast Fragmentation Beams
}

E. Clément, ${ }^{\mathrm{a}, 1}$ A. Görgen, ${ }^{\mathrm{a}}$ W. Korten, ${ }^{\mathrm{a}}$ A. Bürger, ${ }^{\mathrm{a}, \mathrm{b}}$ A. Chatillon, ${ }^{\mathrm{a}, 2}$ Y. Le Coz, ${ }^{\mathrm{a}, 3}$ Ch. Theisen, ${ }^{\text {a }}$ M. Zielińska, ${ }^{\text {a,c }}$ B. Blank, ${ }^{\text {d P.J. Davies, }}{ }^{\text {e }}$ S.P. Fox, ${ }^{\text {e }}$ J. Gerl, ${ }^{\mathrm{f}}$ G. Georgiev,${ }^{\mathrm{g}, 4}$

S. Grévy, ${ }^{\mathrm{g}}$ J. Iwanicki, ${ }^{\mathrm{c}}$ D.G. Jenkins, ${ }^{\mathrm{e}}$ F. Johnston-Theasby, ${ }^{\text {e }}$ P. Joshi, ${ }^{\mathrm{e}}$ I. Matea, ${ }^{\mathrm{d}}$ P.J. Napiorkowski, ${ }^{\mathrm{c}}$ F. de Oliveira Santos, ${ }^{\mathrm{g}}$ M.G. Pellegriti, ${ }^{\mathrm{g}}$ R. Wadsworth ${ }^{\mathrm{e}}$ ${ }^{\text {a } C E A ~ S a c l a y, ~ D A P N I A / S P h N, ~ F-91191 ~ G i f-s u r-Y v e t t e, ~ F r a n c e ~}$

${ }^{\mathrm{b}}$ Helmholtz-Institut für Strahlen- und Kernphysik, Universität Bonn, D-53115 Bonn, Germany

${ }^{\mathrm{c}}$ Heavy Ion Laboratory, Warsaw University, Warsaw, PL-02097, Poland

d CEN Bordeaux-Gradignan, Université Bordeaux I-CNRS/IN2P3, F-33175 Gradignan Cedex, France

e Department of Physics, University of York, York, YO10 5DD, United Kingdom

${ }^{\mathrm{f}}$ Gesellschaft für Schwerionenforschung, D-64291 Darmstadt, Germany g GANIL, BP-5027, F-14076 Caen Cedex, France

\begin{abstract}
A new setup has been designed to perform Coulomb excitation experiments with fragmentation beams at intermediate energy and to measure at the same time conversion electrons from isomeric states populated in the fragmentation reaction. The newly designed setup is described and experimental results from a first experiment are shown. Radioactive even-even nuclei in the mass region $A \simeq 70$ close to the $N=Z$ line were Coulomb excited after fragmentation of an intense primary ${ }^{78} \mathrm{Kr}$ beam and selection in flight with the LISE3 spectrometer at GANIL. The $\gamma$ rays emitted after Coulomb excitation were detected in an array of four large segmented HPGe clover detectors in a very close geometry. The scattered ions were identified in a stack of highly segmented annular silicon detectors combined with a time-of-flight measurement using beam tracking detectors. Conversion electrons from isomeric $0_{2}^{+}$states decaying via electric monopole transitions were detected in an array of segmented cooled silicon detectors surrounding a telescope of plastic scintillators. Reduced transitions probabilities $B\left(E 2 ; 0_{1}^{+} \rightarrow 2_{1}^{+}\right)$were deduced for several stable and radioactive nuclei.
\end{abstract}

Key words: Coulomb excitation, electromagnetic transition probability, conversion-electron spectroscopy, E0 transitions PACS: 25.70.De, 29.30.Dn, 27.50+e

\footnotetext{
1 Present address: CERN, CH-1211 Geneva 23, Switzerland 2 Present address: CEA-DIF/DPTA/SPN, B.P. 12, F-91680 Bruyères-le-Châtel, France

3 Present address: CEA Cadarache, DEN/DTN/SMTM, F13108 Saint-Paul-lez-Durance, France

4 Present address: CSNSM, IN2P3-CNRS, F-91405 Orsay, France
}

\section{Introduction}

A new device is presented for the combined spectroscopy of prompt $\gamma$ rays and isomeric conversion electrons after reactions using fast beams from a fragmentation facility. It has been developed for the physics case of nuclei in the mass region $A \simeq 70$ close to the $N=Z$ line, where low-lying $0^{+}$states related to shape coexistence and shape isomerism are very 
common. Such low-lying $0^{+}$states have been observed experimentally in the even-even Ge isotopes from $A=68$ to 78 , in the Se isotopes from mass $A=$ 70 to 82 , and in the $\mathrm{Kr}$ isotopes from mass $A=72$ to 84 [1].

The $N=Z$ nuclei ${ }^{68}$ Se and ${ }^{72} \mathrm{Kr}$ are also of major importance for the rapid proton capture (rp) nucleosynthesis process on the neutron-deficient side of the nuclear chart [2]. Both nuclei are thought to be waiting points in the rp process: the synthesis of heavier elements can only proceed after $\beta$ decay of rather long-lived isotopes or the sequential capture of two protons. Nuclear structure information of low-lying isomeric states is also an important input parameter for simulations of the rp process [3].

Nuclei in the middle of the $f p$ shell have a large quadrupole deformation in their ground state, and adding or removing a few nucleons can change the nuclear shape dramatically. The self-conjugate nucleus ${ }^{72} \mathrm{Kr}$ is predicted to have an oblate deformation in its ground state [4-7], while the heavier $\mathrm{Kr}$ isotopes were shown to have prolate ground-state deformation and excited states with oblate shape [8]. The first experimental evidence for this shape coexistence was the observation of low-lying $0_{2}^{+}$states [9]. A similar scenario is expected for the light Se isotopes. However, the situation is less clear experimentally, since no low-lying $0_{2}^{+}$state has been observed in ${ }^{68} \mathrm{Se}$.

If a low-lying $0^{+}$state in ${ }^{68} \mathrm{Se}$ is located below or just above the first $2^{+}$state, its decay occurs (exclusively or predominantly) via an $E 0$ transition, i.e. through the emission of a conversion electron. The excited $0^{+}$state then becomes meta-stable with a lifetime of the order of a few to several hundred nanoseconds, depending on the overlap of the wave functions of the two $0^{+}$states. If the nuclei are produced in a high-energy fragmentation reaction $(E>$ $40 \mathrm{AMeV}$ ), they have a high probability of being fully stripped of their atomic electrons. In this case the $E 0$ decay is blocked (if the excitation energy of the excited $0^{+}$state is insufficient for internal pair creation), and the decay can only proceed once the nuclei have regained their atomic (inner-shell) electrons, e.g. after the implantation in a detector.

Intermediate-energy Coulomb excitation after fragmentation is a well-known technique used to measure the reduced transition probability $B\left(E 2 ; 0_{1}^{+} \rightarrow 2_{1}^{+}\right)$for exotic nuclei far from stability, which gives a first indication on the collectivity of the nucleus [10]. Specific equipment has been designed at GSI [11], RIKEN [12], MSU [10], and previously at GANIL [13] for such measurements. Taking advantage of the high beam energy, thick targets can be used to increase the interaction probability. Coulomb excitation events can be separated from other reaction mechanisms by selecting only small scattering angles and thus large impact parameters. Contrary to Coulomb excitation at low energy, where multiple-step excitation is possible, single-step excitations dominate at energies well above the Coulomb barrier, usually simplifying the data analysis. The existence of a low-lying (metastable) $0_{2}^{+}$state can, however, influence the measured Coulomb excitation cross sections and has to be taken into account explicitly. This effect is particularly important if the fragmentation products, which are to be excited in the electromagnetic field of a heavy target nucleus, have been produced with a certain probability in the meta-stable state. In that case the isomeric ratio of the beam has to be measured simultaneously with the Coulomb excitation probability in order to extract $B(E 2)$ values (see e.g. the discussion in Ref. [14]).

Consequently a new device was designed to perform Coulomb excitation experiments of fragmentation products at intermediate energies with the LISE3 spectrometer [15] at GANIL. In addition to the standard detection systems for the tracking and identification of the incident and scattered ions, and for the $\gamma$ rays emitted in-flight after Coulomb excitation, a dedicated setup was installed to measure conversion electrons emitted from unscattered beam particles in silicon detectors after implantation of the ions in a plastic scintillator. In this way the probability of the fragmentation products to be in an isomeric state could be measured and taken into account for the determination of $B(E 2)$ values obtained from the Coulomb excitation process.

\section{Experimental procedure and description of the setup}

The exotic nuclei were produced by the fragmentation of an intense primary ${ }^{78} \mathrm{Kr}^{33+}$ beam at $70.1 A \mathrm{MeV}$, delivered by the coupled GANIL cyclotrons, on a natural $\mathrm{Ni}$ production target located between the two superconducting solenoids of the SISSI device [16]. The target of $125 \mu \mathrm{m}$ thickness was inclined by $25^{\circ}$ and followed by a carbon stripper foil of $10 \mathrm{mg} / \mathrm{cm}^{2}$. The reaction products of interest were selected and purified in the LISE3 spectrometer [15], operated in achromatic mode 


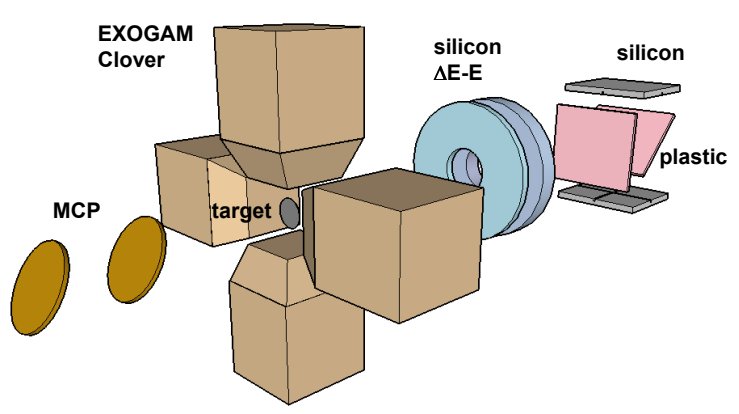

Fig. 1. Schematic view of the experimental setup (not to scale).

with a Be degrader of $215 \mu \mathrm{m}$ thickness. The thickness of both target and degrader was optimized for the production of nuclei on the $N=Z$ line. With a primary beam intensity of $2.4 e \mu \mathrm{A}$ the intensity of the secondary ${ }^{68}$ Se beam on the Coulomb excitation target was $\sim 100$ particles per second. The secondary beam energy was around $40 \mathrm{AMeV}$, depending slightly on the setting of the spectrometer. The composition of the cocktail beam was regularly measured with reduced intensity using a silicon telescope placed before the Coulomb excitation setup.

The complete setup is illustrated schematically in Fig. 1 and described in more detail in the following sections. The associated electronics was based on an analog VXI-VME system and used the standard data-acquisition system of the GANIL facility.

\section{Beam tracking}

The incoming particles were tracked by a set of two micro-channel plate detectors (MCP) [17] of $65 \times 65 \mathrm{~mm}^{2}$ size, measuring secondary electrons produced in a thin emissive foil. The time difference between the first detector and the radio frequency of the cyclotrons provided the time of flight (ToF). The distance between the two detectors was $25 \mathrm{~cm}$ and the second detector was located $29 \mathrm{~cm}$ upstream from the target. Both detectors have a horizontal and vertical position resolution of about $1 \mathrm{~mm}$, allowing the reconstruction of the position and the incident angle of the incoming particles on the target. In this way it was ensured that all nuclei were Coulomb excited under the same conditions. The reconstructed beam spot on the target and the distribution of incident angles are shown in Fig. 2. The beam profile is characterized by a quasi-Gaussian distribution with a horizontal and vertical width of
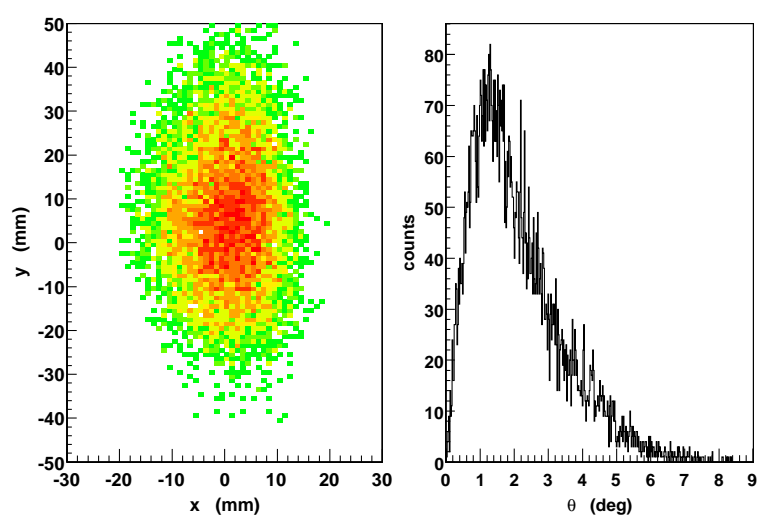

Fig. 2. Beam profile of the ${ }^{68}$ Se projectiles on the ${ }^{208} \mathrm{~Pb}$ target reconstructed from the MCP beam tracking detectors (left) and distribution of the incident angles of the projectiles on the target (right).

$\sigma_{\mathrm{h}}=6 \mathrm{~mm}$ and $\sigma_{\mathrm{v}}=15 \mathrm{~mm}$, respectively, and a mean incident angle of $2^{\circ}$.

\section{Detection of prompt $\gamma$ rays}

The nuclei in the cocktail beam were Coulomb excited on a thick ${ }^{208} \mathrm{~Pb}$ target of $220 \mathrm{mg} / \mathrm{cm}^{2}$ thickness placed in the center of an array of four large segmented clover detectors from the EXOGAM array [18]. Each detector comprises four HPGe crystals and each crystal is segmented into four sectors. The detectors were mounted without BGO escapesuppression shields due to mechanical constraints. The distance between the front face of the detectors and the target was $50 \mathrm{~mm}$. An absolute photo-peak efficiency of $9 \%$ for $1.3 \mathrm{MeV} \gamma$ rays was measured using a ${ }^{60} \mathrm{Co}$ source. The Doppler correction of the $\gamma$ rays which were emitted in flight by the projectile was performed on an event-by-event basis using the position information of the EXOGAM clover detectors and the velocity vector of the outgoing particles.

\section{Detection of $\gamma$ rays from long-lived isomers}

The setup can also be used to measure $\gamma$ rays of isomeric states with lifetimes longer than the time of flight through the spectrometer, which is $\sim 1.2 \mu \mathrm{s}$. In this configuration, the standard silicon telescope of the LISE beam line is introduced about $1 \mathrm{~m}$ upstream from the Coulomb excitation setup. The incident ions are in this way identified by a $\Delta \mathrm{E}-\mathrm{ToF}$ measurement, and they are sufficiently degraded in energy after passing through the Si detectors to be stopped and implanted in the ${ }^{208} \mathrm{~Pb}$ target, which is 


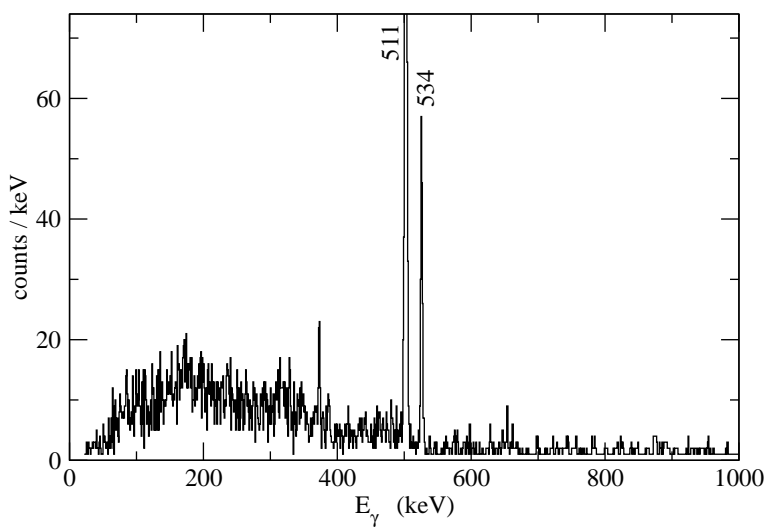

Fig. 3. Spectrum showing the long-lived $9 / 2^{+}$isomer $\left(T_{1 / 2}=960 \mathrm{~ns}\right)$ in ${ }^{69} \mathrm{Se}$ after implantation of the ions into the thick secondary target.

surrounded by the highly efficient array of four EXOGAM clover detectors. This technique can be used to search for long-lived $\gamma$-decaying isomers in exotic nuclei. Furthermore, known isomeric $\gamma$ rays can be used to identify nuclides unambiguously in the $\Delta \mathrm{E}$ ToF plot. This was for example possible using the $9 / 2^{+}$isomer in ${ }^{69} \mathrm{Se}$, which has a half live of $T_{1 / 2}=$ $960 \mathrm{~ns}$ and decays via a $534 \mathrm{keV} M 2$ transition [19]. The $\gamma$-ray spectrum of ${ }^{69}$ Se nuclei implanted into the ${ }^{208} \mathrm{~Pb}$ target is shown in Fig. 3 .

\section{Detection of scattered particles}

At high beam energies a pure electromagnetic excitation process can only be ensured by selecting large impact parameters corresponding to small scattering angles. The scattered particles were detected and identified in a telescope of two highly segmented double-sided annular silicon strip detectors placed $42 \mathrm{~cm}$ downstream from the ${ }^{208} \mathrm{~Pb}$ target. The two silicon detectors were segmented into 16 concentric rings on the front side and 16 azimuthal sectors on the back. Both detectors had active surfaces with inner and outer radii of 11 and $35 \mathrm{~mm}$, respectively. The first silicon detector covered scattering angles between $1.5^{\circ}$ and $4.76^{\circ}$ in the laboratory frame, ensuring that the impact parameter was always greater than $17 \mathrm{fm}$ for all nuclei. With a thickness of $147 \mu \mathrm{m}$ the first detector provided a measure of the energy loss $\Delta E$, while the second silicon detector of $300 \mu \mathrm{m}$ thickness determined the residual energy $E$. The knowledge of the time of flight, $\Delta E$, and $E$ allows the unambiguous identification of all outgoing fragments in mass and atomic number. The contribution of nuclear

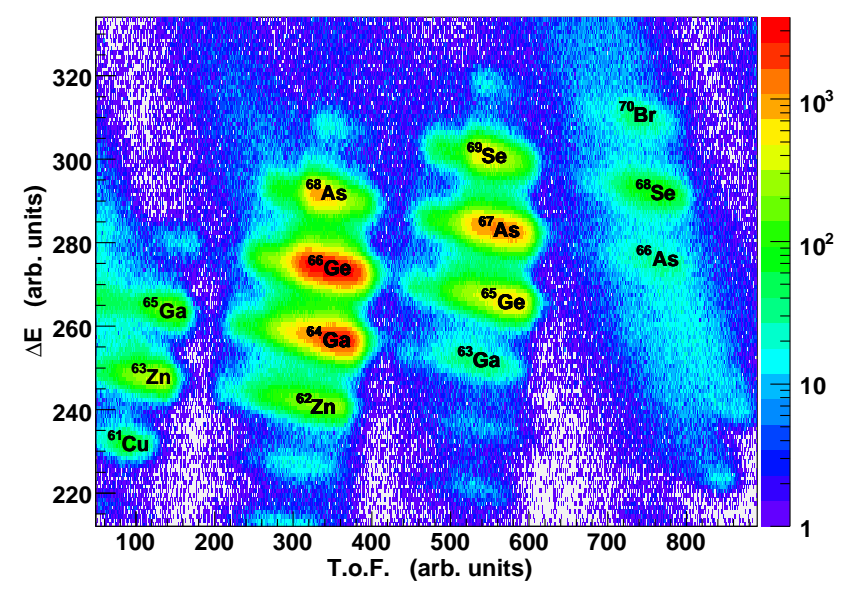

Fig. 4. Identification matrix of the energy loss in the silicon detector against the time of flight between the cyclotron RF and the beam tracking detector. The setting of LISE3 was optimized for ${ }^{68} \mathrm{Se}$.

reactions in the secondary target such as transfer can thus be excluded. An identification plot of the energy loss $\Delta E$ and the time of flight between the cyclotron RF and the first MCP detector is shown in Fig. 4. Nuclides with the same isospin have a similar time of flight through the spectrometer, but can clearly be separated by the $\Delta E$ measurement.

\section{Isomer detection system}

Non-interacting beam particles which passed through the central hole in the annular silicon detectors were detected in a telescope of two plastic scintillators of $300 \mu \mathrm{m}$ thickness, placed $62 \mathrm{~cm}$ behind the ${ }^{208} \mathrm{~Pb}$ target. The second scintillator was tilted by $45^{\circ}$ with respect to the beam axis. The thickness of the scintillators was chosen such that the ions were implanted in the center of the second scintillator. Due to the limited energy resolution, a $Z$ identification via a $\Delta E-E$ measurement could not be achieved. However, the detectors were used to determine the total number of implanted nuclei. The exact beam composition was determined in regular intervals of a few hours by introducing a standard stack of silicon detectors into the beam upstream from the secondary target. In this way the absolute rate of the different nuclides incident on the target could be monitored.

The second scintillator was placed in the center of a modified version of the BEST array [20], originally designed for the spectroscopy of transfermium isotopes. Two alcohol-cooled silicon detectors of $1 \mathrm{~mm}$ thickness and divided into four quadrants each were 
facing the tilted surfaces of the second scintillator as illustrated in Fig. 1. Each silicon detector had an active surface of $50 \times 50 \mathrm{~mm}^{2}$. The distance between the two Si detectors was $50 \mathrm{~mm}$. The beam particles arrived on the secondary target at $40 \mathrm{AMeV}$ fully stripped of their atomic electrons. Most of the ions regain electrons only when they are implanted in the plastic scintillator. Ions arriving in an excited $0^{+}$ state which decays exclusively via an $E 0$ transition are therefore trapped; the decay can only proceed after implantation, no matter how long the lifetime or the flight time through the spectrometer is. Even if there is an $E 2$ branch depopulating the $0^{+}$state, the partial lifetime can become longer than the time of flight. The array of silicon detectors around the implantation scintillator is therefore well suited to search for low-lying excited $0^{+}$states, populated in the fragmentation reaction, by detecting the conversion electrons of the $E 0$ decay.

The upper silicon detector suffered from lowenergetic $\delta$ electrons produced by the ions passing through the first scintillator. The lower silicon detector is mostly shielded by the tilted scintillator, resulting in much cleaner spectra. In cases of longlived isomers decaying by low-energy $\gamma$ rays, it was possible to detect these $\gamma$ rays in the silicon detectors, for example the $5.7 \mu \mathrm{s}$ isomer in ${ }^{73} \mathrm{As}$, decaying by a $360 \mathrm{keV} \gamma$ ray.

\section{Commissioning with stable ${ }^{78} \mathrm{Kr}$ and ${ }^{72} \mathrm{Ge}$ beams}

The commissioning of the setup was performed using ${ }^{78} \mathrm{Kr}$ and ${ }^{72} \mathrm{Ge}$ beams. The $B\left(E 2 ; 0_{1}^{+} \rightarrow 2_{1}^{+}\right)$ values are known and very large. The first excited state in ${ }^{72} \mathrm{Ge}$ is a $0_{2}^{+}$state at $691 \mathrm{keV}$, making ${ }^{72} \mathrm{Ge}$ the ideal case to test the setup for the detection of isomeric conversion electrons. Instead of using the primary ${ }^{78} \mathrm{Kr}$ beam directly, a secondary beam of ${ }^{78} \mathrm{Kr}$ was produced under conditions similar to those of the later measurement with radioactive nuclides. The beam was tracked by the MCP detectors and the velocity vector of the incident beam particles reconstructed. The $\gamma$ rays emitted after Coulomb excitation of the beam were detected in coincidence with a scattered particle identified as ${ }^{78} \mathrm{Kr}$ in the annular silicon telescope. The Doppler correction was performed using the angular information of both the annular silicon detector and the segmented outer electrodes of the clover detectors. The calculated value of the beam velocity after passing the ${ }^{208} \mathrm{~Pb}$ target

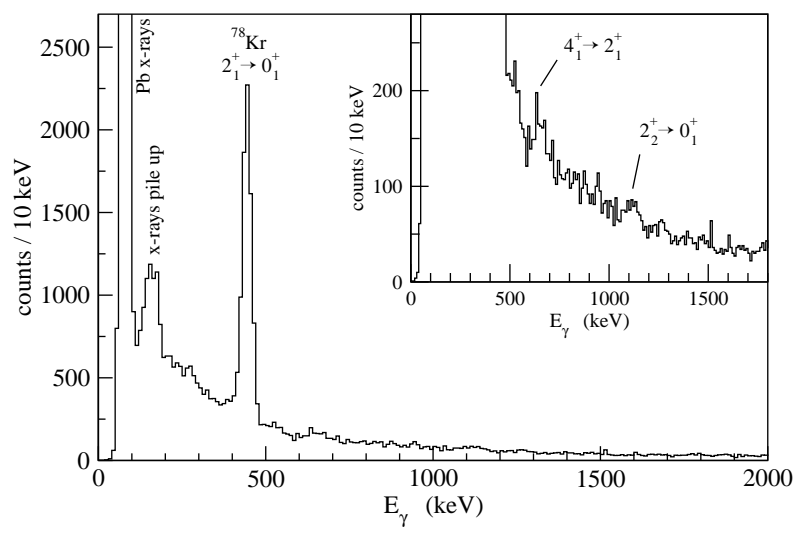

Fig. 5. Prompt $\gamma$-ray spectrum in coincidence with scattered ${ }^{78} \mathrm{Kr}$ projectiles. The inset shows the same spectrum on an enlarged scale. At low energy the spectrum is dominated by $\mathrm{Pb}$ x-rays from the target.

was used since the lifetime of the $2_{1}^{+}$state in ${ }^{78} \mathrm{Kr}$ is longer than the time of flight through the target. For nuclei with shorter lifetimes, the velocity at mid-target was applied. Due to the large collectivity of ${ }^{78} \mathrm{Kr}$, the $2_{1}^{+}$state is strongly populated. The prompt Doppler-corrected $\gamma$-ray spectrum gated on ${ }^{78} \mathrm{Kr}$ is presented in Fig. 5. Since there is only one $\gamma$ ray emitted in the vast majority of Coulomb excitation events, a large fraction of the background was suppressed by requiring that only one germanium crystal gave signals. Indications for the population of the $2_{2}^{+}$state and two-step processes populating the $4_{1}^{+}$state are presented in the inset of Fig. 5. The $3^{-}$state in ${ }^{208} \mathrm{~Pb}$ at $2.6 \mathrm{MeV}$ was also populated, but the peak is too spread out in energy to be seen in the spectrum which was Doppler corrected for ${ }^{78} \mathrm{Kr}$. A resolution of $30 \mathrm{keV}$ was achieved for the $2_{1}^{+} \rightarrow 0_{1}^{+}$ transition at $455 \mathrm{keV}$, mainly limited by the velocity spread in the target and the opening angle of the clover detector segments.

In the same way, the prompt Doppler-corrected $\gamma$-ray spectrum gated on ${ }^{72} \mathrm{Ge}$ was obtained. As previously discussed, ${ }^{72} \mathrm{Ge}$ has a first excited state of spin-parity $0^{+}$. As the nuclei are fully stripped, the ${ }^{72} \mathrm{Ge}$ nuclei arrive with a certain percentage in the isomeric state; this isomeric ratio has to be taken into account in the Coulomb excitation analysis. The unscattered ${ }^{72} \mathrm{Ge}$ ions were implanted into the scintillator where they regained their atomic electrons allowing the decay of the $0_{2}^{+}$state to proceed by conversion electrons. The spectrum obtained with the lower silicon detector is presented in Fig. 6. It shows very clearly the $0_{2}^{+} \rightarrow 0_{1}^{+} E 0$ decay of $691 \mathrm{keV}$ in ${ }^{72} \mathrm{Ge}$. The spectrum is gated on the time of flight for 


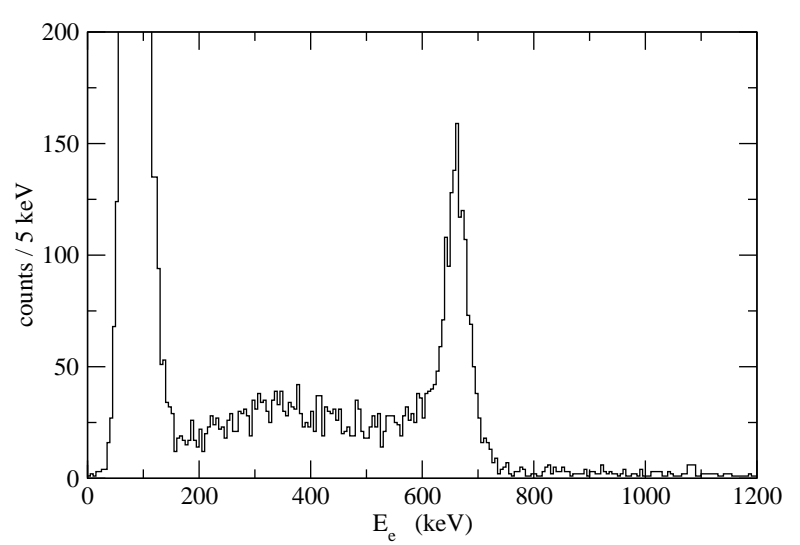

Fig. 6. Conversion-electron spectrum in coincidence with ${ }^{72} \mathrm{Ge}$ nuclei showing the $E 0$ decay of the first excited $0^{+}$ state. The peak at low energy is due to atomic electrons.

nuclei with $T_{z}=4$. However, the energy resolution of the scintillator was insufficient to resolve the ${ }^{72} \mathrm{Ge}$ fragments from neighboring ${ }^{74} \mathrm{As}$ and ${ }^{70} \mathrm{Ga}$. Since the beam composition is known, the isomeric ratio can be determined, and it was found that about $3 \%$ of the incident ${ }^{72} \mathrm{Ge}$ ions arrive in the isomeric $0_{2}^{+}$ state.

The measurements with ${ }^{78} \mathrm{Kr}$ and ${ }^{72} \mathrm{Ge}$ have shown that the setup is well suited to perform Coulomb excitation experiments at intermediate energy and measure simultaneously the ratio of ions arriving in an isomeric $0^{+}$state. This allows determining $B(E 2)$ values for exotic nuclei in the presence of low-lying $0^{+}$states.

\section{Measurements with Radioactive Beams}

As the next step the spectrometer setting was optimized for several radioactive nuclei with mostly known $B(E 2)$ values. The prompt Dopplercorrected $\gamma$-ray spectra in coincidence with ${ }^{66} \mathrm{Ge}$, ${ }^{62} \mathrm{Zn}$ and ${ }^{68} \mathrm{Se}$ are presented in Fig. 7. Due to electronics and data acquisition problems in this part of the experiment, only a small fraction of the recorded events were correlated, and the signals from the outer contacts of the clover detectors were not coded at all, leading to a severe loss of statistics and a degraded energy resolution. The $2_{1}^{+} \rightarrow 0_{1}^{+}$transitions are nevertheless clearly visible for ${ }^{66} \mathrm{Ge}$ and ${ }^{62} \mathrm{Zn}$. In the case of ${ }^{68} \mathrm{Se}$, no significant peak is visible above background level at the $2^{+}$energy of $853 \mathrm{keV}$. An upper limit of 40 counts can be extracted from the spectrum gated on ${ }^{68}$ Se for the $2_{1}^{+} \rightarrow 0_{1}^{+}$transition.

In order to determine unknown $B(E 2)$ values and to minimize systematic errors, nuclei with well

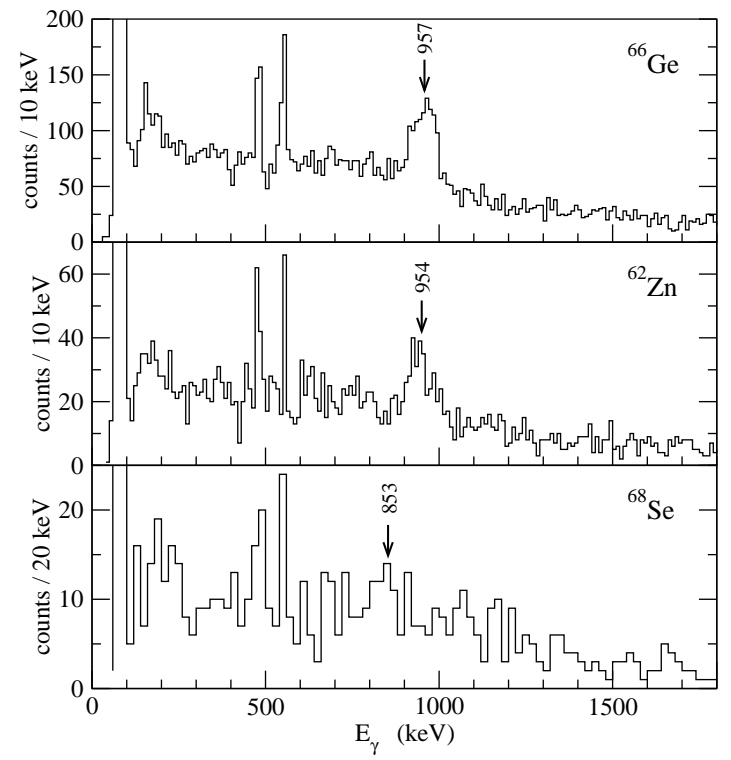

Fig. 7. Prompt Doppler corrected $\gamma$ spectra for ${ }^{66} \mathrm{Ge}$ (top), ${ }^{62} \mathrm{Zn}$ (center), and ${ }^{68} \mathrm{Se}$ (bottom). The energies of the $2_{1}^{+} \rightarrow 0_{1}^{+}$transitions are marked by arrows. Other peaks are due to $\mathrm{x}$-rays from the ${ }^{208} \mathrm{~Pb}$ target, the pile-up of $\mathrm{x}$-rays, and the $511 \mathrm{keV}$ annihilation line, which appears as a doublet due to the Doppler correction.

known $B(E 2)$ values are used as a reference to normalize the unknown values. For this procedure, the $\gamma$-ray intensities must be corrected for angular distribution effects and for the energy dependence of the $\gamma$-ray efficiency. As the $B(E 2)$ of ${ }^{66} \mathrm{Ge}$ and ${ }^{62,64} \mathrm{Zn}$ nuclei are known, they could be used as a reference to determine an upper limit for the $B(E 2)$ value for ${ }^{68} \mathrm{Se}$. The data from the ${ }^{78} \mathrm{Kr}$ and ${ }^{72} \mathrm{Ge}$ measurements could not be used for normalization as the experimental conditions were not identical due to the electronics and data acquisition problems. The $B(E 2)$ values were extracted using an approximation of the exact Coulomb excitation cross sections at relativistic energies via the so-called "recoil correction" method developed by Alder and Winther [21]. It was shown [22] that this correction is the best approximation in the energy range between 20 and $40 A \mathrm{MeV}$ used in the present experiment.

The results of the present measurement are summarized in table 1 . The systematics of $B\left(E 2 ; 2_{1}^{+} \rightarrow\right.$ $0_{1}^{+}$) values for the $\mathrm{Zn}, \mathrm{Ge}$, and Se chains is presented in Fig. 8. The values derived from the present measurement are shown as full symbols. The results are fully compatible with the known $B(E 2)$ values for ${ }^{64} \mathrm{Zn}$ and ${ }^{66} \mathrm{Ge}$ (open symbols). The value for ${ }^{62} \mathrm{Zn}$, however, is found to be smaller than the literature value based on the measured lifetime of $4.2(3) \mathrm{ps}$ 
Table 1

Deduced $B\left(E 2 ; 2^{+} \rightarrow 0^{+}\right)$values for ${ }^{62} \mathrm{Zn},{ }^{64} \mathrm{Zn}$, and ${ }^{66} \mathrm{Ge}$. The results are compared to literature values. Only an upper limit can be deduced for ${ }^{68} \mathrm{Se}$.

\begin{tabular}{ccc} 
Nucleus & \multicolumn{2}{c}{$B\left(E 2 ; 0_{1}^{+} \rightarrow 2_{1}^{+}\right)\left[e^{2} \mathrm{~b}^{2}\right]$} \\
& Nuclear Data & this work \\
\hline${ }^{62} \mathrm{Zn}$ & $0.124(9)[23]$ & $0.074(15)$ \\
${ }^{64} \mathrm{Zn}$ & $0.149(5)[24]$ & $0.165(21)$ \\
${ }^{66} \mathrm{Ge}$ & $0.099(19)[23]$ & $0.093(12)$ \\
${ }^{68} \mathrm{Se}$ & & $\leq 0.157$ \\
\hline
\end{tabular}

[25]. Consequently, only the values for ${ }^{64} \mathrm{Zn}$ and ${ }^{66} \mathrm{Ge}$ were used for the normalization. The resulting $B\left(E 2 ; 2_{1}^{+} \rightarrow 0_{1}^{+}\right)$value for ${ }^{62} \mathrm{Zn}$ is $10.2(21)$ Weisskopf units. For ${ }^{68} \mathrm{Se}$, an upper limit of $B\left(E 2 ; 2_{1}^{+} \rightarrow\right.$ $\left.0_{1}^{+}\right)<19$ W.u. is extracted.

The data illustrate the strong collectivity of the isotopes at mid-shell, which decreases toward the shell closures at 28 and 50. A pronounced irregularity is observed for the Se isotopes, which show an unexpected staggering at $N=36$ and 38 [26]. However, the relevant lifetimes have been re-measured recently and the data indicate that ${ }^{70} \mathrm{Se}$ is less collective than previously thought [27]. The upper limit for the $B(E 2)$ value of ${ }^{68}$ Se supports the interpretation of a smooth and symmetric behavior with a maximum of collectivity at $N=40$ and 42 , which is decreasing with neutron number. The new value for ${ }^{62} \mathrm{Zn}, B\left(E 2 ; 0_{1}^{+} \rightarrow 2_{1}^{+}\right)=0.074(15) e^{2} \mathrm{~b}^{2}$, suggests that also for the $\mathrm{Zn}$ isotopes the collectivity decreases stronger with neutron number than previously thought.

The array of plastic scintillators and silicon detectors was used to search for the predicted $0_{2}^{+}$ shape isomer in ${ }^{68} \mathrm{Se}$ by detecting conversion electrons emitted from the ${ }^{68} \mathrm{Se}$ ions implanted into the plastic scintillator. Assuming that a similar fraction of ${ }^{68} \mathrm{Se}$ ions is produced in an isomeric state as for ${ }^{72} \mathrm{Ge}(\sim 3 \%)$, one would expect to observe about 400 conversion electrons in the lower silicon detector, ignoring the upper one which suffers from a higher background of $\delta$ electrons. Since no sign of an $E 0$ transition was observed in the spectrum conditioned on the ToF for $T_{z}=0$ nuclei and an energy loss for $Z \approx 34$, the presence of a $0_{2}^{+}$state as the first excited state in ${ }^{68} \mathrm{Se}$ can be excluded. Even a $0_{2}^{+}$state situated slightly above the $2_{1}^{+}$state should have been observed due to the expected long partial lifetime in that case. If a low-lying $0_{2}^{+}$state exists in ${ }^{68} \mathrm{Se}$, it can be presumed to lie well above the $2_{1}^{+}$ state, to which it can decay through $\gamma$ rays. This

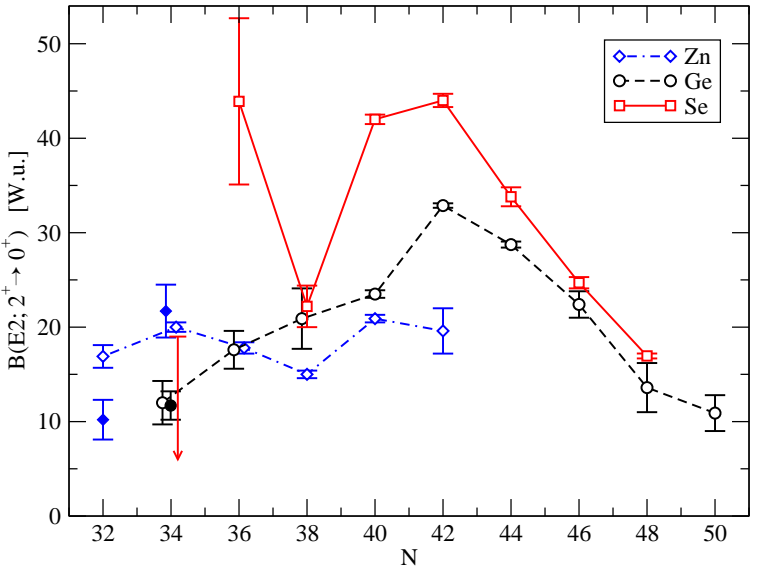

Fig. 8. Systematics of the $B\left(E 2 ; 2_{1}^{+} \rightarrow 0_{1}^{+}\right)$values for $\mathrm{Zn}$, $\mathrm{Ge}$, and Se isotopes. Values taken from Ref. [23] are shown as open, data from the present work as full symbols. The data points are slightly displaced horizontally for clarity of the presentation.

is the case in neighboring ${ }^{70} \mathrm{Se}$, where an excited $0_{2}^{+}$state has been observed at $2011 \mathrm{keV}$ excitation energy, decaying to the $2_{1}^{+}$state at $945 \mathrm{keV}[26]$.

In order to search for possible isomers decaying by $\gamma$ rays, a stack of silicon detectors was inserted into the beam line before the ${ }^{208} \mathrm{~Pb}$ target, which was used to identify the ions event by event and to degrade their energy so that they were implanted in the $\mathrm{Pb}$ target. With this method the $960 \mathrm{~ns}$ isomer in ${ }^{69}$ Se was observed (see Fig. 3). Again, the spectrum conditioned on ${ }^{68}$ Se showed no sign of an isomer, so that the presence of a $\gamma$-decaying isomer with a lifetime longer than $\sim 1 \mu \mathrm{s}$ in ${ }^{68}$ Se can be excluded. The experimental results for ${ }^{68} \mathrm{Se}$ and in particular the non-observation of a low-lying $0_{2}^{+}$state stress the importance of further experimental studies for the understanding of the predicted shape coexistence in the light Se isotopes.

\section{Summary}

A new setup was developed for intermediateenergy Coulomb excitation combined with spectroscopy of isomeric conversion electrons. Coulomb excitation of stable and radioactive beams in the $A \sim 70$ region at $40 A \mathrm{MeV}$ has been performed following the fragmentation of an intense ${ }^{78} \mathrm{Kr}$ primary beam of $70 A \mathrm{MeV}$. The setup was tested using secondary ${ }^{78} \mathrm{Kr}$ and ${ }^{72} \mathrm{Ge}$ beams. The latter served also to test the setup for the detection of isomeric conversion electrons from the $0_{2}^{+}$state in ${ }^{72} \mathrm{Ge}$. It was found that $3 \%$ of the secondary beam arrived in the isomeric $0_{2}^{+}$state, the decay of which is 
blocked during the flight through the spectrometer. $B(E 2)$ values were measured for several radioactive secondary beams. The $B(E 2)$ value for ${ }^{62} \mathrm{Zn}$ was found to be lower than the literature value. Only an upper limit for the $B(E 2)$ value could be derived for the $N=Z$ nucleus ${ }^{68} \mathrm{Se}$. The existence of a low-lying, long-lived $0_{2}^{+}$state with a lifetime longer than $\sim 1 \mu \mathrm{s}$ in ${ }^{68}$ Se could be excluded.

\section{Acknowledgments}

We would like to thank the technical staff at GANIL for excellent support during the preparation and running of the experiment. This work has been supported in part by the European Community FP6 - Structuring the ERA - Integrated Infrastructure Initiative - contract EURONS No. RII3-CT-2004506065 , by the Polish State Committee for Scientific Research under contract No. 2 P03B 007 25, by the German BMBF under contract 06BN-109, and the UK EPSRC.

\section{References}

[1] R.B. Firestone, V.S. Shirley, C.M. Baglin, S.Y.F. Chu, J. Zapkin, eds., Table of Isotopes (Wiley, New York, 1996)

[2] H. Schatz et al., Phys. Rep. 294, 167 (1998)

[3] S.E. Woosley et al., ApJS 151, 75, (2004)

[4] R. Bengtsson, Nuclear Structure of the Zirconium Region (eds. J. Eberth, R.M. Meyer, and K. Siestemich Springer-Verlag, 1988)

[5] A. Petrovici, K.W. Schmid, Amand Faessler, Nucl. Phys. A 665, 333 (2000)

[6] M. Bender, P. Bonche, P.-H. Heenen, Phys. Rev. C 74, $024312(2006)$

[7] M. Girod, http://www-phynu.cea.fr/science_en_ligne /carte_potentiels_microscopiques/carte_potentiel_ nucleaire.htm and to be published

[8] E. Clément et al., Phys. Rev. C 75, 054313 (2007)

[9] E. Bouchez et al., Phys. Rev. Lett. 90, 082502 (2003)

[10] T. Glasmacher et al., Ann. Rev. Nucl. Part. Sci. 48, 1 (1998)

[11] H.J. Wollersheim et al., Nucl. Inst. and Meth. A 537, 637 (2005)

[12] T. Motobayashi et al., Phys. Lett. 346B, 9 (1995)

[13] O. Sorlin et al., Phys. Rev. Lett. 88, 092501 (2002)

[14] A. Gade et al., Phys. Rev. Lett. 95, 022502 (2005) and Phys. Rev. Lett. 96, 189901(E) (2006)

[15] R. Anne, D. Bazin, A.C. Mueller, J.C. Jacmart, M. Langevin, Nucl. Inst. and Meth. A 257, 215 (1987)

[16] Eric Baron, Jean Gillet, Michel Ozille, Nucl. Inst. and Meth. A 362, 90 (1995)

[17] O.H. Odland et al., Nucl. Inst. and Meth. A 378, 149 (1996)

[18] F. Azaiez et al., Acta Phys. Hung. A 11, 159 (2000)
[19] K.R. Pohl, D.F. Winchell, J.W. Arrison, D.P. Balamuth, Phys. Rev. C 51, 519 (1995)

[20] A. Chatillon et al., Eur. Phys. J. A 30, 397 (2006)

[21] Aage Winther and Kurt Alder, Nucl. Phys. A 319, 518 (1979)

[22] C.A. Bertulani, A.E. Stuchbery, T.J. Mertzimekis, A.D. Davies, Phys. Rev. C 68, 044609 (2003)

[23] S. Raman, C.W. Nestor Jr., P. Tikkanen, Atomic Data and Nucl. Data Tables 78, 1 (2001)

[24] J. Leske et al., Phys. Rev. C 71, 034303 (2005)

[25] N.J. Ward et al., J. Phys. G 7, 815 (1981)

[26] J. Heese et al., Z. Phys. A 325, 45 (1986)

[27] J. Ljungvall et al., to be published 